\title{
Perfil clínico y microbiológico de las lesiones por minas antipersonal en el Hospital Pablo Tobón Uribe, Medellín, 2003-2005
}

\author{
Ana Cristina Restrepo, Jaime Alberto López \\ Laboratorio de Microbiología, Hospital Pablo Tobón Uribe, Medellín, Colombia
}

\begin{abstract}
Introducción. El problema de las lesiones por minas antipersonal crece cada día en el mundo, pero poco se conoce en Colombia sobre las características clínicas y microbiológicas de esas lesiones.

Objetivos. Determinar las características clínicas y microbiológicas de las lesiones por minas antipersonal en los pacientes atendidos en el Hospital Pablo Tobón Uribe, durante los años 2003 a 2005.

Materiales y métodos. Se revisaron las historias clínicas de 151 pacientes hospitalizados por lesiones causadas por minas antipersonal, entre el 1ํ de enero de 2003 y el 31 de julio de 2005, en un hospital universitario de alta complejidad de Medellín, Colombia.

Resultados. Todos los pacientes eran de sexo masculino, con edad promedio de 24 años y $94,7 \%$ de ellos eran militares. La estancia hospitalaria promedio fue de 20 días y la mortalidad hospitalaria de $4 \%$. El 66,8\% presentaba lesiones múltiples, 88\% compromiso de las extremidades y las amputaciones afectaron a $52,3 \%$ de los pacientes.

Se realizaron 313 cultivos, 68,7\% positivos, para un total de 386 microorganismos aislados. Las bacterias más frecuentes en el primer cultivo fueron Pseudomonas aeruginosa y Escherichia coli, y en los cultivos posteriores, P. aeruginosa y Enterococcus faecalis. El 93,4\% de los pacientes recibió tratamiento antibiótico empírico; el más utilizado fue ampicilina/sulbactam.

Conclusiones. Las características clínicas y microbiológicas de los pacientes atendidos están relacionadas con su ocupación y los complejos mecanismos de daño de las minas. Un mejor conocimiento de dichas características permitirá diseñar estrategias de manejo y tratamientos empíricos de acuerdo con la epidemiología local.
\end{abstract}

Palabras clave: heridas y traumatismos, traumatismo múltiple, traumatismos por explosión, amputación, infección.

Clinical and microbiological profiles of anti-personnel mine injuries in the Medellín area of northwestern Colombia

Introduction. The counts of antipersonnel mine injuries are increasing worldwide, but in Colombia, documentation of the clinical and microbiological characteristics of these lesions is lacking.

Objectives. To determine the clinical and microbiological characteristics of antipersonnel mine injuries for patients admitted for treatment during a 2 and a half year period.

Materials and methods. The medical records were reviewed for 151 patients hospitalized with injuries caused by antipersonnel mines. The admissions occurred between January $1^{\text {st }} 2003$ and July $31^{\text {st }} 2005$ at a highly specialized university hospital, the Pablo Tobón Uribe Hospital in Medellín, Colombia.

Results. All patients were male, mean age 24 years old, and all but 8 of them military. The length of stay averaged 20 days and hospital mortality $4 \%$. Multiple lesions occurred in $66.8 \%$ of the cases, $88.0 \%$ of the injuries affected the limbs, and amputations were mandated in $52.3 \%$ of patients. A total of 313 specimens were cultured--68.7\% of cultures were positive, and 386 microorganisms were identified. The most prevalent bacteria in the first culture were Pseudomonas aeruginosa and Escherichia coli. In subsequent cultures, $P$. aeruginosa and Enterococcus faecalis appeared. Most of the patients (93.4\%) received standard antibiotic therapy, ampicillin/sulbactam.

Conclusions. The clinical and microbiological characteristics of patients were related to their military occupation and the complex injuries produced by the mines. Knowledge of these characteristics will permit the design of better management strategies and empirical treatments.

Key words: Wounds and injuries, multiple trauma, blast injuries, amputation, infection. 
El problema de contaminación por minas antipersonal es un fenómeno mundial. Se calcula que hay entre 65 y 120 millones de minas antipersonal diseminadas en 70 países de todo el mundo (1-7). La Asamblea Mundial de Salud reconoció el tema como un problema de salud pública en 1998 (8).

El uso de minas antipersonal es sólo una parte del conflicto armado que ha afectado a Colombia por más de 40 años. Son utilizadas en zonas de combate y alrededor de los campamentos, para defender posiciones estratégicas, lugares de retención de secuestrados y como armas indiscriminadas de terror (9).

Colombia tiene el mayor número de víctimas en el mundo y cada año hay algo más de mil víctimas nuevas (7). Además, es el país con el mayor número de minas sembradas en su suelo. Afectan el $46 \%$ de los municipios del país y a 31 de los 32 departamentos. El departamento de Antioquia registra el mayor número de casos en el país: $19,8 \%$ del total (10).

Las minas antipersonal causan lesiones múltiples mediantecomplejos mecanismos detrauma, heridas de alta contaminación, estancias hospitalarias prolongadas, incapacidades y secuelas, sumadas a los altos costos de atención $(11,12)$, pero las estadísticas relacionadas con estas lesiones en Colombia son limitadas.

El objetivo general de la presente investigación fue determinar las características clínicas y microbiológicas de las lesiones por minas antipersonal en los pacientes hospitalizados y egresados del Hospital Pablo Tobón Uribe, durante 31 meses de estudio.

\section{Materiales y métodos}

Mediante un estudio descriptivo y retrospectivo, se incluyeron todos los pacientes registrados al egreso por traumatismos ocasionados en operaciones de guerra, causados por esquirlas de minas terrestres antipersonal, según la Clasificación Internacional de Enfermedades y problemas relacionados con la salud (CIE 10) (13), del primero de enero de 2003 al 31 de julio de 2005, en el Hospital Pablo

Correspondencia:

Ana Cristina Restrepo, Calle 78B № 69-240, Medellín, Colombia.

Teléfono: (54) 459 286; fax: (54) 417955

acrestrepo@hptu.org.co

Recibido: 22/09//09; aceptado:29/03/10
Tobón Uribe de Medellín, hospital universitario de carácter general de alto nivel de complejidad, con 270 camas. No se incluyeron los pacientes con traumatismos ocasionados por esquirlas de minas terrestres que no fueron hospitalizados ni los reingresos hospitalarios por complicaciones crónicas de lesiones por minas antipersonal.

La información se obtuvo de la revisión de las historias clínicas, de la base de datos del seguimiento microbiológico del Laboratorio de Microbiología del Hospital Pablo Tobón Uribe y de la base de datos de los cultivos positivos registrados en el programa Whonet.

Se identificaron las características demográficas de los pacientes, los tiempos transcurridos desde la lesión hasta su atención en el hospital y hasta el primer procedimiento quirúrgico, el sitio anatómico comprometido y el tipo de lesiones en las extremidades: heridas de piel, fractura cerrada, fractura abierta, quemadura y amputación. Se clasificaron como múltiples, cuando para el sitio anatómico o tipo de lesión implicaban dos o más opciones.

También, se estableció la estancia hospitalaria y su estado al alta, los cultivos realizados, el tipo de cultivo y origen de la muestra. Además, se determinó la etiología microbiana de las infecciones asociadas a las lesiones o adquiridas en el hospital, la sensibilidad antibiótica de los microorganismos cultivados, los antibióticos empíricos y terapéuticos empleados como consecuencia de las lesiones por minas antipersonal, y los microorganismos cultivados.

Los cultivos e identificaciones microbiológicas se realizaron según los estándares reconocidos (14) y los estudios de sensibilidad antimicrobiana de acuerdo con lo establecido por el Clinical Laboratory and Standards Institute (CLSI) de los Estados Unidos (15). Con respecto al análisis de la sensibilidad antimicrobiana de las bacterias cultivadas, en el caso de cepas de igual género y especie que demostraban el mismo patrón de sensibilidad y aisladas en el mismo paciente, sólo se tomó una de ellas, para el análisis final correspondiente.

Laclasificación de infección hospitalaria relacionada con las lesiones causadas por minas antipersonal, se hizo de acuerdo con las recomendaciones de los Centers for Disease Control and Prevention (CDC) de 1996 (16). Los datos obtenidos se analizaron empleando el programa Excel® de Windows 2003. 


\section{Resultados}

Se encontraron 213 egresos hospitalarios por esquirlas de mina terrestre o bomba, código Y362 según la CIE 10 (13). Al revisar las historias clínicas se excluyeron 62 pacientes que habían presentado heridas en operaciones de guerra y que habían sido causadas por otros mecanismos diferentes a las minas antipersonal, como explosiones con bombas, granadas y misiles, entre otros.

Se incluyeron en el estudio 151 egresos hospitalarios por minas antipersonal. Durante el 2003, se atendieron en promedio dos pacientes mensuales, cuatro pacientes por mes en el 2004 y 11 en el 2005.

Todos los pacientes incluidos en el estudio ingresaron por el Servicio de Urgencias, todos eran de sexo masculino, con edad promedio de 24 años (rango, 18 a 37 años), $143(94,7 \%)$ de ellos eran militares y $8(5,3 \%)$ civiles.

La estancia hospitalaria promedio fue de 20 días (entre uno y 109 días). Seis pacientes fueron remitidos a otra institución. La mortalidad hospitalaria fue de $4 \%$ (seis pacientes) y, en promedio, se presentó al cuarto día de estancia, $y$ todos los fallecidos presentaban amputaciones y lesiones múltiples de las extremidades.

Se encontraron datos del tiempo de evolución desde que ocurrió la lesión hasta el ingreso a urgencias en 145 casos, con un promedio de 12 horas (rango, 1 a 144 horas). Se sometieron a cirugía $132(87,4 \%)$ pacientes luego del ingreso a urgencias; en ocho de ellos no hay registro del tiempo transcurrido desde la lesión. El tiempo de evolución hasta la cirugía fue de 15 horas, en promedio (rango, 1 a 125 horas).

En 50 (33,2\%) pacientes las lesiones se presentaron en un solo sitio anatómico; los 101 restantes $(66,8 \%)$ presentaban lesiones múltiples (dos o más sitios anatómicos). El cuadro 1 muestra la distribución por sitio de lesión. Los sitios anatómicos más afectados fueron las extremidades en $133(88 \%)$ de los pacientes, $126(83,4 \%)$ con lesiones en extremidades inferiores y $51(33,8 \%)$ en extremidades superiores. El tipo de lesión en las extremidades fue múltiple (dos o más) en 78 pacientes. El comportamiento del tipo de lesiones en las extremidades se presenta en el cuadro 2.

Aunque superadas en frecuencia por las heridas de piel en las extremidades, las amputaciones afectaron $79(52,3 \%)$ del total de pacientes incluidos en el estudio.
Cuadro 1. Distribución por sitio de lesiones causadas por minas antipersonal, Hospital Pablo Tobón Uribe, Medellín, 2003 a julio 2005.

\begin{tabular}{lcr}
\hline Sitio de lesión & $\mathbf{n}$ & $\%$ \\
\hline Cabeza y cuello & 42 & 27,8 \\
Ojos & 39 & 25,8 \\
Tórax & 10 & 6,6 \\
Abdomen & 10 & 6,6 \\
Extremidad superior derecha & 25 & 16,6 \\
Extremidad superior izquierda & 35 & 23,2 \\
Extremidad inferior derecha & 94 & 62,3 \\
Extremidad inferior izquierda & 96 & 63,6 \\
Genitales & 10 & 6,6 \\
\hline n=151 pacientes & &
\end{tabular}

$=151$ pacientes

Cuadro 2. Tipo de lesiones causadas por minas antipersonal en extremidades, Hospital Pablo Tobón Uribe, Medellín, 2003 a julio 2005.

\begin{tabular}{lrr}
\hline Tipo de lesión en extremidades & $\mathbf{n}$ & $\%$ \\
\hline Heridas de piel & 81 & 60,9 \\
Amputación & 79 & 59,4 \\
Fractura abierta & 41 & 30,8 \\
Fractura cerrada & 28 & 21,1 \\
Quemadura & 9 & 6,8 \\
\hline
\end{tabular}

$\mathrm{n}=133$

En 87 (57,6\%) se obtuvo muestra para cultivo y la primera muestra se obtuvo en promedio a las 122 horas (rango, 8 a 573, con una mediana de 93 horas) de ocurrida la lesión y 110 horas después del ingreso a urgencias (rango, 3 a 570, con una mediana de 77 horas).

Se realizaron 313 cultivos, 3,5 cultivos por paciente, $82,1 \%$ para microorganismos aerobios y $17,9 \%$ para anaerobios. El número máximo de cultivos fue de 13 para un solo paciente. En cuanto al origen de la muestra, $97 \%$ se obtuvieron de las extremidades, con mayor frecuencia de la extremidad inferior derecha.

De los 313 cultivos realizados, 215 (68,7\%) fueron positivos y se identificaron 386 microorganismos, 1,8 aislamientos por cultivo positivo (cuadro 3). De los 87 pacientes en los cuales se tomó muestra para cultivo, 75 tuvieron al menos un resultado positivo. Los bacilos Gram negativos fueron los microorganismos más frecuentes (68\%), seguidos por los cocos Gram positivos (29\%), bacilos Gram positivos (1,6\%), cinco cepas de anaerobios y sólo una levadura. Los 15 tipos de gérmenes más frecuentes constituyeron el 86,3\% del total, 11 de los cuales fueron bacilos Gram negativos y cuatro cocos Gram positivos, como se muestra en el cuadro 4. Los aislamientos de 
Cuadro 3. Número de microorganismos identificados por cultivo en lesiones por minas antipersonal, Hospital Pablo Tobón Uribe, Medellín, 2003 a julio 2005.

\begin{tabular}{lccccc}
\hline Cultivos & Negativo & Un germen & Dos gérmenes & Más de dos gérmenes & Total \\
\hline Total & 98 & 98 & 68 & 49 & 313 \\
Porcentaje & 31,3 & 31,3 & 21,7 & 15,7 & 100 \\
\hline
\end{tabular}

otros 25 tipos de microorganismos fueron poco frecuentes. La sensibilidad a los antibióticos de los microorganismos cultivados se presenta en los cuadros 5 y 6 .

En 141 pacientes $(93,4 \%)$ se administró tratamiento antibiótico empírico, 74 con monoterapia y 67 con terapia combinada. Los antibióticos empíricos utilizados como monoterapia fueron: ampicilina/ sulbactam (52 pacientes), ciprofloxacina (8 pacientes), cefalotina (6 pacientes), cefazolina (6 pacientes), ceftriaxona ( 1 paciente) e imipenem (1 paciente).

Las combinaciones antibióticas empíricas más frecuentes fueron: ciprofloxacina-clindamicina, cefalotina-gentamicina, ciprofloxacina-ampicilina/ sulbactam y cefalotina-metronidazol. En 70 pacientes $(46 \%)$ fue necesario el cambio o la adición de antibióticos terapéuticos dirigidos a los microorganismos cultivados. Los más frecuentes fueron: ciprofloxacina, vancomicina, ampicilina/ sulbactam, aztreonam y cefazolina. Se presentaron infecciones hospitalarias relacionadas con las lesiones causadas por minas antipersonal en 35 $(23,2 \%)$ pacientes, en las cuales se aislaron 90 microorganismos, y los cinco más frecuentes

Cuadro 4. Microorganismos aislados en lesiones por minas antipersonal, Hospital Pablo Tobón Uribe, Medellín, 2003 a julio 2005 .

\begin{tabular}{lrr}
\hline Germen aislado & $\mathbf{n}$ & $\%$ \\
\hline Pseudomonas aeruginosa & 51 & 13,2 \\
Escherichia coli & 37 & 9,6 \\
Enterococcus faecalis & 34 & 8,8 \\
Enterobacter cloacae & 31 & 8,0 \\
Aeromonas hydrophila & 28 & 7,3 \\
Staphylococcus epidermidis & 24 & 6,2 \\
Serratia marcescens & 23 & 6,0 \\
Klebsiella pneumoniae & 21 & 5,4 \\
Enterobacter aerogenes & 18 & 4,7 \\
Staphylococcus aureus & 16 & 4,1 \\
Klebsiella oxytoca & 14 & 3,6 \\
Morganella morganii & 12 & 3,1 \\
Enterococcus faecium & 11 & 2,8 \\
Stenotrophomonas maltophilia & 7 & 1,8 \\
Acinetobacter baumannii & 6 & 1,6 \\
Otros & 53 & 13,7 \\
Total & 386 & 100 \\
\hline
\end{tabular}

(Pseudomonas aeruginosa, Escherichia coli, Enterococcus faecalis, Staphylococcus epidermidis y Enterobacter cloacae) fueron responsables de $52 \%$ de las infecciones hospitalarias.

\section{Discusión}

El aumento sostenido de los ingresos de pacientes con lesiones por minas antipersonal durante el período del estudio, se relacionacon la consolidación del hospital como centro de referencia en el manejo de trauma de alta complejidad y el fortalecimiento de convenios de atención con las Fuerzas Militares de Colombia.

Esta razón explica también la edad, el sexo y la condición militar de los pacientes incluidos. La mortalidad hospitalaria es similar a la reportada en otros trabajos publicados, $3,8 \%$ y $7 \%$ en pacientes procedentes de conflictos armados de Afganistán, Camboya y Sudán $(17,18)$. No hay datos disponibles para comparar la seriedad de las lesiones o los recursos hospitalarios de los sitios de atención. El Monitor de Minas Terrestres, sitio oficial de Landmine Monitor Core Group, reportó un tiempo promedio de 12 horas para que los sobrevivientes de minas antipersonal lleguen a un hospital regional en Colombia (9), el mismo tiempo promedio encontrado en este estudio, y que se relaciona con dificultades para el rescate y transporte de los lesionados desde el sitio del evento hasta el hospital.

El porcentaje de pacientes sometidos a cirugía fue muy alto $(87,4 \%)$, pero no está disponible el dato en otros estudios consultados. El tiempo transcurrido desde la lesión hasta el inicio de la cirugía fue de 15 horas, en promedio, reconocido como un factor de riesgo para la infección de las heridas, pues es crítico para el control de la contaminación y la escisión de tejido muerto $(19,20)$.

De manera similar a los reportes sobre heridas de guerra, los pacientes presentaron con mayor frecuencia lesiones de piel, músculo esquelético de las extremidades y amputaciones (21). En el presente estudio hubo una frecuencia mayor de lesión primaria en las extremidades (88\%), y en 
Cuadro 5. Sensibilidad antibiótica de los cocos Gram positivos aislados en lesiones por minas antipersonal, Hospital Pablo Tobón Uribe, Medellín, 2003 a julio 2005.

\begin{tabular}{lccc}
\hline Antibiótico & Staphylococcus epidermidis & Staphylococcus aureus & Enterococcus faecalis \\
\hline Ciprofloxacina & 57 & 40 \\
Clindamicina & 64 & 40 \\
Eritromicina & 43 & 40 \\
Gentamicina & 60 & 14 \\
Oxacillina & 31 & 40 \\
Rifampicina & 79 & 100 \\
Tetraciclina & 58 & 100 \\
Trimetoprim/sulfametoxasol & 43 & 100 & 100 \\
Vancomicina & 100 & 100 & 100 \\
Linezolid & 100 & 100 & 100 \\
Ampicillina & & & \\
\hline
\end{tabular}

Cuadro 6. Sensibilidad antibiótica de los bacilos Gram negativos aislados en lesiones por minas antipersonal, Hospital Pablo Tobón Uribe, Medellín, 2003 a julio 2005.

\begin{tabular}{|c|c|c|c|c|c|c|c|c|}
\hline Antibiótico & $\begin{array}{c}\text { Pseudomonas } \\
\text { aeruginosa }\end{array}$ & $\begin{array}{c}\text { Escherichia } \\
\text { coli }\end{array}$ & $\begin{array}{c}\text { Enterobacter } \\
\text { cloacae }\end{array}$ & $\begin{array}{c}\text { Aeromonas } \\
\text { hydrophila }\end{array}$ & $\begin{array}{c}\text { Serratia } \\
\text { marcescens }\end{array}$ & $\begin{array}{c}\text { Enterobacter } \\
\text { aerogenes }\end{array}$ & $\begin{array}{c}\text { Klebsiella } \\
\text { pneumoniae }\end{array}$ & $\begin{array}{c}\text { Klebsiella } \\
\text { oxytoca }\end{array}$ \\
\hline Amikacina & 96 & 100 & 93 & 100 & 100 & 100 & 100 & 100 \\
\hline \multicolumn{9}{|l|}{ Ampicillina/ } \\
\hline sulbactam & & 88 & 21 & 0 & 0 & 50 & 56 & 86 \\
\hline & & 53 & & & & & & \\
\hline Aztreonam & 79 & 100 & 93 & 100 & 100 & 100 & 100 & 86 \\
\hline Cefazolina & & 94 & 7 & 0 & 0 & 10 & 56 & 57 \\
\hline Cefepime & 92 & 100 & 100 & 100 & 100 & 100 & 100 & 100 \\
\hline \multicolumn{9}{|l|}{ Cefoperazona/ } \\
\hline sulbactam & 87 & 100 & 93 & 83 & 100 & 100 & 89 & 100 \\
\hline Cefoxitin & & 82 & 14 & 67 & 17 & 20 & 56 & 86 \\
\hline Ceftazidima & 96 & 100 & 93 & 100 & 100 & 100 & 100 & 100 \\
\hline Ceftriaxona & & 100 & 93 & 100 & 100 & 100 & 100 & 100 \\
\hline Ciprofloxacina & 92 & 82 & 93 & 92 & 100 & 100 & 100 & 100 \\
\hline Gentamicina & 92 & 94 & 93 & 100 & 100 & 100 & 100 & 100 \\
\hline Imipenem & 100 & 100 & 100 & 100 & 100 & 100 & 100 & 100 \\
\hline \multirow{2}{*}{\multicolumn{9}{|c|}{ Piperacillina/ }} \\
\hline & & & & & & & & \\
\hline tazobactam & 90 & 100 & 93 & 100 & 100 & 100 & 89 & 100 \\
\hline \multirow{2}{*}{$\begin{array}{l}\text { Trimetoprim/ } \\
\text { sulfametoxasol }\end{array}$} & & & & & & & & \\
\hline & & 82 & 93 & 92 & 83 & 90 & 100 & 100 \\
\hline
\end{tabular}

cabeza y cuello $(27,8 \%)$, comparada con $26 \%$ y $10 \%$, respectivamente, reportadas por Champion H. et al. en heridas de guerra (21), explicada por el mecanismo de trauma propio de las minas antipersonal, modelo I de Coupland, asociadas a detonadores de presión al pisarlas. La frecuencia de lesiones de tórax y abdomen causadas por múltiples fragmentos de minas activadas cerca de la víctima (modelo II de Coupland) fue similar (17). La frecuencia de la amputación traumática por minas antipersonal de una o más extremidades $(52,3 \%)$, está en el rango de $30 \%$ a $85 \%$ reportado en la literatura $(12,22,23)$.

No conocemos estudios indexados relacionados con la obtención de muestras para cultivos en heridos por minas antipersonal que permitan comparación. Sin embargo, el porcentaje de cultivos positivos y el número de aislamientos por cultivo obtenidos, corroboran la frecuencia de infecciones y su etiología polimicrobiana. Considerando que en $86,2 \%$ de los pacientes en quienes se tomó muestra para cultivo ( $49,6 \%$ del total) se obtuvo, al menos, un resultado positivo, debe recomendarse la búsqueda activa de la etiología microbiana de las lesiones.

Las bacterias cultivadas de las lesiones fueron comparables con los reportes de la literatura en heridas similares (19). P. aeruginosa fue el principal aislamiento, seguido por E. coli. Staphylococcus aureus, reconocido como la primera causa de infección de heridas traumáticas (20), ocupó el décimo lugar, tal vez como consecuencia de la 
terapia antibiótica empírica empleada. Vale la pena destacar la presencia en el quinto lugar de Aeromonas hydrophila, una bacteria muy poco reportada en los países de climas templados, pero de amplia distribución en ambientes tropicales y que concuerda con las características climáticas y geográficas de Colombia. Se estima que los anaerobios pueden ser demostrados en 30\% de las heridas de guerra (19); en este estudio el porcentaje de cultivos para anaerobios practicados fue bajo $(17,9 \%$ del total) y fueron positivos sólo en 9\%. Estos resultados pueden estar relacionados con las condiciones de recolección y transporte de las muestras.

En la sensibilidad de las bacterias cultivadas a los antibióticos se debe tener en cuenta que se analizaron en conjunto las bacterias adquiridas en la comunidad y en el ambiente hospitalario. El número de cepas no repetidas no permitía hacer un análisis comparativo de ambos grupos. La clasificación de la infección como intrahospitalaria en este tipo de lesiones resulta tentativa, debido a la variabilidad en el tiempo transcurrido entre la lesión y la toma de muestra para el cultivo (3 a 573 horas), lo cual dificulta determinar la flora contaminante al momento del ingreso de los pacientes.

En cuanto a los cocos Gram positivos, se destaca que $S$. aureus (diez cepas cultivadas en total, cuatro de ellas intrahospitalarias) sólo fue sensible a la oxacilina en $40 \%$ de los casos. De nuevo, la terapia empírica empleada podría haber seleccionando la flora microbiana. El $100 \%$ de las cepas estudiadas de $S$. aureus fueron sensibles a trimetoprim/sulfametoxasol y tetraciclina, los cuales podrían emplearse como terapia de consolidación. $S$. epidermidis ocupó el cuarto lugar y, como era de esperarse, sólo fueron sensibles a la oxacilina el $30 \%$ de las cepas. Como en la mayoría de los casos que involucran este tipo de bacterias, el discriminar entre colonización, contaminación e infección resulta problemático.

En el Hospital Pablo Tobón Uribe, antes de proceder a reportar este tipo de aislamientos, se consideran: el tipo de la muestra, el método de recolección, el resultado de la coloración de Gram y el cultivo en los medios sólidos o líquidos.

En cuanto a los principales bacilos Gram negativos, las alternativas antibióticas de mayor a menor cubrimiento fueron: imipenem (100\%), meropenem $(99,5 \%)$, cefepime $(99 \%)$, ceftazidima y amikacina $(98,6 \%)$, gentamicina $(97,3 \%)$, piperacilina/tazobactam
(96,5\%), ciprofloxacina (94,8\%), aztreonam (94,7\%) y cefoperazona/sulbactam (94\%). Como puede observarse, no existieron diferencias importantes entre la mayoría de los antibióticos estudiados. De los antibióticos sin actividad contra $P$. aeruginosa, sólo la ceftriaxona con una sensibilidad del $99 \%$ de los bacilos Gram negativos y el trimetoprim/ sulfametoxasol con $91,4 \%$, serían opciones de tratamiento, ya que las sensibilidades a cefoxitin $(48,4 \%)$, ampicilina/sulbactam (43\%) y cefazolina (32\%) fueron muy bajas.

Debe recordarse que en presencia de Serratia marcescens, E. cloacae, Enterobacter aerogenes y $A$. hydrophila, no se recomienda el empleo de antibióticos betalactámicos, por la posibilidad de inducir la producción de betalactamasas tipo AmpC, lo cual limita el uso de ceftriaxona a pesar de su excelente actividad in vitro contra bacilos Gram negativos.

Por la naturaleza de las lesiones por minas antipersonal y el tiempo que transcurre desde su ocurrencia hasta la atención médica, la mayoría se consideran infectadas (20), lo cual explica la utilización empírica de antibióticos en $93,4 \%$ de los pacientes de este estudio.

Es importante señalar que el principal antibiótico empleado de esta forma, la ampicilina/sulbactam, no era efectivo para las principales especies bacterianas cultivadas. Debido a la variedad de los posibles microorganismos involucrados en la etiología de las infecciones por este tipo de lesiones, resulta difícil establecer una terapéutica antibiótica que cubra todos los posibles agentes etiológicos y que no genere problemas relacionados con costos, efectos adversos o resistencia microbiana.

El esquema empírico sugerido por el Comité Internacional de la Cruz Roja para heridos por minas antipersonal es penicilina más metronidazol (19). De acuerdo con los hallazgos en este estudio, este esquema no es aplicable a las circunstancias locales. Se requiere de equipos interdisciplinarios con entrenamiento para la atención de los pacientes lesionados con minas antipersonal, y el reconocimiento de las características clínicas y microbiológicas de las lesiones para una terapia orientada según la epidemiología local. De los resultados obtenidos, se sugiere establecer guías o protocolos de manejo de las lesiones en el sitio de la explosión y en los centros de atención primaria y de referencia, procurar la identificación de la flora presente en las lesiones al ingreso del paciente para orientar el manejo antibiótico empírico o 
terapéutico, y realizar estudios comparativos del uso de antibióticos empíricos para evaluar su efectividad.

\section{Conflicto de intereses}

La presente investigación se realizó como parte del proceso del día a día del Laboratorio de Microbiología y por iniciativa propia; por lo tanto, no subyace ningún interés que pudiera haber intervenido en la metodología o en las conclusiones del trabajo.

\section{Financiación}

Este estudio fue financiado en su totalidad por el Hospital Pablo Tobón Uribe.

\section{Referencias}

1. Comité Internacional de la Cruz Roja. Minas terrestres antipersonal, ¿armas indispensables? Estudio sobre el uso militar y la eficacia de las minas antipersonal. Ginebra: CICR; 1996.

2. Giannou C. Antipersonal landmines: facts, fictions and priorities. BMJ.1997;315:1453-4.

3. Vidal JL. Fundamentos terapéuticos actuales para los heridos de extremidades durante la guerra. Rev Cubana Med Milit.1999;28:31-40.

4. Williams J. Las minas terrestres y su eliminación. Revista Internacional de la Cruz Roja. 1995;130:409-27.

5. Cauderay G. Las minas antipersonal. Revista Internacional de la Cruz Roja.1993;118:289-305.

6. Comité Internacional de la Cruz Roja. Para poner fin a la era de las minas terrestres. América: Panorama y perfiles de los países. Ginebra: CICR; 2004.

7. De Colombia Amigos. Las minas antipersona: el terror que azota a Colombia. Fecha de consulta: 30 de agosto de 2009. Disponible en: http://decolombiaamigos.com/index.p hp? view=article \&catid=37\%3Ainformesdebase $\& i d=53 \% 3 \mathrm{Al}$ as-minas-antipersona-un-drama colombiano\&option=com content\&Itemid=56.

8. Krug E, Ikeda R, Qualls M, Anderson MA, Rosenberg ML, Jackson RJ. Preventing land mine-related injury and disability: a public health perspective. JAMA. 1998;280:465-6.

9. Landmine Monitor. International campaign to ban landmines. Colombia. 2009 Fecha de acceso: 30 de agosto de 2009. Disponible en: http://www.Im.icbl.org/index.php.
10. Vicepresidencia de la República de Colombia. Programa Presidencial de los Derechos Humanos y Derecho Internacional Humanitario. Observatorio de Minas. Fecha de consulta: 30 de agosto de 2009. Disponible en: http:// www.derechoshumanos.gov.co/PNA/estadisticasminas. asp.

11. Vicepresidencia de la República de Colombia. Programa Presidencial de los Derechos Humanos y Derecho Internacional Humanitario. Observatorio de los Derechos Humanos en Colombia. Fecha de consulta: 30 de agosto de 2009. Disponible en: http://derechos humanos.gov.co/ observatorio/.

12. Clasper J, Rew D. Trauma life support in conflict. BMJ. 2003;327:1178-9

13. Organización Mundial de la Salud. Clasificación Estadística Internacional de Enfermedades y Problemas de Salud Conexos (CIE). Décima Revisión (CIE-10). Ginebra: OMS; 1992.

14. American Society for Microbiology. Clinical microbiology procedures. Handbook. Second edition. Washington, D.C.: American Society for Microbiology; 2004.

15. Clinical and Laboratory Standards Institute (CLSI). Performance standards for antimicrobial susceptibility testing; CLSI document M100-S19. Wayne, PA: CLSI; 2005.

16. Garner JS, Jarvis WR, Emori TG, Horan TC, Hughes JM. CDC definitions for nosocomial infections. En: Olmsted RN, editor. APIC Infection Control and Applied Epidemiology: Principles and Practice. St. Louis: Mosby; 1996. p. A1-20.

17. Coupland R, Russbach R. Victims of antipersonal mines: What is being done? Med Glob Surviv. 1994;1:18-22.

18. Wennerstrom M. Injuries associated with landmines and unexploded ordnance-Afghanistan, 1997-2002. JAMA. 2003;290:1846-8.

19. Dufour D, Kromann-Jensen S, Owen-Smith M, Salmela J, Steneng GF, Zetterström B. Surgery for victims of war. Third edition. Ginebra: CICR; 1998.

20. Bowler PG, Buerden BI, Armstrong DG. Wound microbiology and associated approaches to wound management. Clin Microbiol Rev. 2001;14:244-69.

21. Champion H, Bellamy R, Roberts C, Leppaniemi A. A profile of combat injury. J Trauma. 2003;54:S13-9.

22. Brown R, Chaloner E, Mannion S, Cheatle T. 10-year experience of injuries sustained during clearance of antipersonnel mines. Lancet. 2001;358:2048-9.

23. Sulayvani F. Antipersonnel land mines injuries in Dohuk region/northern Iraq. The Middle East Journal of Emergency Medicine. 2001;1:6. 